Proceedings

\title{
Panic buying behavior analysis of COVID-19 related toi- let paper hoarding content on Twitter
}

\author{
Janni Leung ${ }^{1,2}$, Calvert Tisdale ${ }^{1,2}$, Jack Yiu Chak Chung, ${ }^{1,2, *}$, Vivian Chiu ${ }^{1,2}$, Carmen C. W. Lim ${ }^{1,2}$, Gary \\ Chan $^{2}$
}

Publisher's Note: MDPI stays neutral with regard to jurisdictional claims in published maps and institutional affiliations.

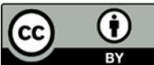

Copyright: $(2020$ by the authors. Submitted for possible open access publication under the terms and conditions of the Creative Commons Attribution (CC BY) license (http://creativecommons.org/licenses/by/4.0/).
1 National Centre for Youth Substance Use Research, The University of Queensland, Brisbane, Australia; cysar@uq.edu.au

2 School of Psychology, The University of Queensland, Brisbane, Australia; reception@psy.uq.edu.au

* Correspondence: yiuchak.chung@uqconnect.edu.au

\begin{abstract}
Aim: The COVID-19 pandemic had increased population-level anxiety and elicited panic buying behaviours across the world. We analysed Tweets related to panic buying of toilet paper during the crisis. Methods: A dataset of $n=255,171$ Tweets were collected and analysed by adopting a grounded theory approach until saturation was met (n=4,081). Results: Five key themes emerged: 1) humour or sarcasm, 2) marketing or profiteering, 3) opinion and emotions, 4) personal experience, and 5) support or information. About half of the Tweets carried negative sentiments. Conclusions: Findings have implications on how the government and relevant stakeholders could monitor and react to public concerns using contemporary social media data.
\end{abstract}

Keywords: COVID-19; pandemic; anxiety; panic buying; social media; psychological phenomena

\section{Introduction}

The Coronavirus disease (COVID-19) has been declared a global pandemic in March 2020. Anxiety and fear were seen particularly with panic buying behaviours across the world [1, 2]. The COVID-19 driven panic buying phenomenon has left supermarket shelves empty for weeks. Panic buying is defined as when consumers purchase an exceptionally large amount of products in the anticipation of a shortage. We have not seen panic buying behaviour to this extent in previous disease outbreaks [3]. The hoarding of a particular household item, toilet paper, has received a lot of public attention in the media, sending shockwaves to demand and supply chains [4].

There are several plausible explanations for the panic buying phenomenon. One of the rational reasons for panic buying behaviours is to minimise potential risks such as running out of supplies. Panic stocking is a natural human response to disasters and emergency crises to regain control of fear [5]. However, prolonged exposure to traumatic events and media coverage on the crises could lead to psychological distress even in the absence of posttraumatic stress disorder (PTSD) [6, 7]. Stockpiling daily necessities may offer comfort over unpredictable circumstances [8]. Herding mentality also account for panic buying, as people are influenced by others to make emotional decisions [9].

Social media was thought to be amplifying the sense of scarcity, with stories about people engaging in hoarding supplies being highlighted. The use of Twitter, a social media platform, has become increasingly widespread 
across populations as a way to reflect their opinions and engage with others [10]. Twitter provides data on public conversations that has demonstrated research utility on public health concerns [11], e.g. on harmful alcohol use of young people [12], to monitor mental health discussions [13], infodemiology studies of the H1N1 pandemic [14, 15].

To understand social media information sharing, qualitative or quantitative research methodologies is often adopted, applying with grounded theory approach, which construct theories based on empirical data, instead of testing a priori hypotheses [16]. This enables generalisation of theories through comprehending concurrent phenomenon, to understand human behaviours and mentality. This study draws on Twitter's data to examine anxiety-related panic buying behaviour during COVID-19 by analysing public opinions and attitudes towards related Tweets.

\section{Methods}

\subsection{Qualitative approach}

This study follows Standards for Reporting Qualitative Research (SRQR) guidelines. We applied a grounded theory approach to develop themes and theories from the dataset using an interpretivist paradigm. Theories behind the panic buying phenomenon were constructed through methodical gathering and analysis of Tweets by coding them into themes. This approach could formulate hypotheses based on existing empirical Tweets. This paradigm was adopted to explain subjective reasons behind phenomenological behaviours [17].

\subsection{Researcher characteristics}

The researchers are based in Australia, aged 25-35, with background in psychology, epidemiology, and statistics. The researchers are not, and do not have acquaintances, who are prolific social mediasts.

\subsection{Sampling strategy}

We have collected 255,171 unique original Tweets using our search terms and excluded 257,090 Tweets that had less than 10 Retweets. A total sample of 4,081 eligible Tweets met our inclusion criteria.

We adopted data saturation approach, a sampling method that estimates the likely number of qualitative data needed to reach saturation for a given study [18]. A random sub-sample of 100 Tweets were selected and classified according to the themes identified. The process was repeated until no new topics were being found. The 10 Tweets with the most Likes and Retweets were selected to represent the most influential Tweets.

\subsection{Data collection}

Original Tweets about COVID-19 related hoarding of toilet paper were collected between 29 Feb and 29 March 2020. The search terms included "toilet paper", "toilet roll", \#toiletpaper, \#toiletpapercrisis, \#ToiletPaperEmergency, \#toiletpapergate, \#toiletpaperwars, \#toiletpaperroll, and \#toiletpaperrolls. Data was collected using an automatic web crawler [19] with Python 3.7.3 on 8 April 2020.

\subsection{Data processing}

Tweets from each of the searches were collected into a csv file, and duplicates were removed. Filters were applied to remove Tweets with less than 10 Retweets. Only text contents of Tweets were collected. 


\subsection{Data analysis}

The present study adopted an approach that is consistent with existing studies on previous infectious disease outbreak [14]. Literature review on Twitter analysis methodologies was first conducted, followed by data collection and screening. Two researchers then reviewed Tweets using data saturation technique. Sentiments of the Tweets were coded by analysing their contents. Sub-themes were developed to assimilate the data, followed by creating themes. This coding scheme was applied when coding the Tweets. Tweets were randomised to code. Coding was terminated when data saturation was met $20 \%$ of consecutive Tweets conveys the same themes, as no new themes or sub-categories emerge.

\section{Results}

\subsection{Tweet characteristics}

Of all the Tweets analysed until saturation reached at $n=500,46 \%$ carried negative sentiments regarding the panic buying phenomena of toilet paper, $13 \%$ carried positive sentiments, with the remaining $31 \%$ having neutral sentiments. Tweets were 3.5 times more likely to carry negative sentiments than positive sentiments. The majority (87\%) of Tweets had 10 to 99 Retweets (Table 1). The 5 mostly shared Tweets had been Retweeted more than 1,000 times. In addition, $88 \%$ of Tweets received 0 to 499 likes, and $6 \%$ of Tweets received more than 1,000 likes.

\subsection{Influential Tweets}

Among the most influential Tweets (Table S1), half of them contained negative sentiments, with expressions of frustration or anger over the lack of toilet rolls, or sarcasm, humour, and confusion towards the phenomenon. Some of the highly influential Tweets were supportive and provided update information about the panic buying situation.

Table 1. Descriptive statistics of Retweets and Likes of the original Tweets .

\begin{tabular}{|c|c|c|c|c|}
\hline & \multicolumn{2}{|c|}{ Frequency of original Tweets } & \multicolumn{2}{|c|}{ Totality of engagement } \\
\hline & $\mathbf{n}$ & $\%$ & $\Sigma$ & $\%$ \\
\hline \multicolumn{5}{|l|}{ Retweets } \\
\hline Total & 500 & $100 \%$ & 53,141 & $100 \%$ \\
\hline \multicolumn{5}{|l|}{ By number of Retweets } \\
\hline 10-99 RTs & 433 & $86.6 \%$ & 11,679 & $24.7 \%$ \\
\hline 100-999 RTs & 62 & $12.4 \%$ & 15,371 & $34.2 \%$ \\
\hline 1000-1999 RTs & 3 & $0.6 \%$ & 3,774 & $8.4 \%$ \\
\hline 2000-4999 RTs & 1 & $0.2 \%$ & 3,895 & $9.0 \%$ \\
\hline $5000+$ RTs & 1 & $0.2 \%$ & 18,422 & $23.8 \%$ \\
\hline \multicolumn{5}{|l|}{ Likes } \\
\hline Total & 500 & $100 \%$ & 272,630 & $100 \%$ \\
\hline \multicolumn{5}{|l|}{ By number of likes } \\
\hline $0-499$ & 422 & $88.4 \%$ & 55,710 & $20.4 \%$ \\
\hline $500-999$ & 31 & $6.2 \%$ & 21,545 & $7.9 \%$ \\
\hline 1000-4999 & 24 & $4.8 \%$ & 47,728 & $17.5 \%$ \\
\hline 5000-9999 likes & 1 & $0.2 \%$ & 6,216 & $2.3 \%$ \\
\hline 10,000-29,999 likes & 0 & $0 \%$ & 0 & $0 \%$ \\
\hline $30,000+$ likes & 2 & $0.4 \%$ & 141,431 & $51.9 \%$ \\
\hline
\end{tabular}

3.3. Themes 
Five prominent themes were identified from the data, which include: 1) Humour or sarcasm; 2) Opinion and emotion; 3) Personal experience; 4) Support or information; and 5) Monetary (see Table 2).

\subsubsection{Theme 1: Humour or sarcasm}

Humour and sarcasm were the most common themes of the toilet paper related Tweets (26\%). These Tweets were amusing or eliciting humour sarcastically. This included humour using alternatives as toilet paper, mocking the insanity of panic buying, making jokes about how future generations would view this frenzy. For example:

Since toilet paper now available at our nearby supermarket, we are cancelling our newspaper subscription.

\subsubsection{Theme 2: Opinion and emotion}

Many of the Tweets carried strong opinions and beliefs with feelings and emotions. Anger was the most dominant emotion (24\%), followed by having sceptical (13\%) or unconcerned (6\%) opinions. Twitter users expressed anger and frustration towards the insufficiency of toilet paper. Some other users felt sceptical about the necessity of stockpiling toilet paper. Some were unconcerned about the situation. For example:

Does the \#coronavirus make you shit yourself to death then? Why the hell is everyone stockpiling \#toiletpaper?!

\subsubsection{Theme 3: Personal experience}

This theme included Tweets about events that individuals experienced, negatively or positively. Some of these Tweets referred to negative personal experience of failing to obtain toilet paper $(10 \%)$ and unpleasant shopping experience $(6 \%)$. Only a few expressed joys over obtaining adequate toilet paper $(3 \%)$ and selfless acts of helping others (1\%). For example:

I only have six spare toilet rolls and one in use. Living life on the edge! \#panicbuyinguk

\subsubsection{Theme 4: Support or information}

This theme included Tweets providing support and information regarding panic buying of toilet rolls. They included Tweets of shared information relating to the panic buying situation such as where to obtain toilet paper $(15 \%)$, news from media (7\%) and providing support $(7 \%)$. Three sub-themes of support, information and media were identified in the coding process. Some Twitter users shared information such as website links and articles on where to obtain toilet paper. Other users shared news regarding panic buying phenomenon whereas some provided support or expressed they have received support from others. For example:

Women fight over toilet paper at Australia supermarket amid \#coronavirus fears. https://wwww.shorturl.at/w]LY2 
Table 2. Themes, with counts, and stats of sentiment.

\begin{tabular}{|c|c|c|}
\hline Themes and sub-themes identified & Definition & Percentage \\
\hline 1. Humour or sarcasm & Aimed to be amusing, comical, or to illicit humour & $26 \%$ \\
\hline 2. Opinion and emotions & Carried emotions, views and beliefs & \\
\hline Anger & Anger and frustration over others' panic buying behaviour & $24 \%$ \\
\hline Sceptical & Scepticism over the need to panic buy & $13 \%$ \\
\hline Unconcerned & Tweets that express unconcern over panic buying & $6 \%$ \\
\hline 3. Personal experience & Tweets about events that individuals experienced & \\
\hline \multicolumn{3}{|l|}{ Negative } \\
\hline Insufficient supply & Inability to obtain toilet paper & $10 \%$ \\
\hline Unpleasant shopping experience & Negative experience of shopping for toilet paper & $6 \%$ \\
\hline \multicolumn{3}{|l|}{ Positive } \\
\hline Access to toilet paper & Able to gain access to toilet paper & $3 \%$ \\
\hline Altruism & Tweets related to people helping others on toilet paper & $1 \%$ \\
\hline 4. Support or information & Tweets that provided support or information & \\
\hline Information & Tweets that provided information & $15 \%$ \\
\hline Media & Tweets by news outlets & $7 \%$ \\
\hline Support & Tweets about providing or receiving support & $7 \%$ \\
\hline 5. Monetary & $\begin{array}{c}\text { Money-related, such as trying to promote a product or profiteering through toi- } \\
\text { let paper }\end{array}$ & $4 \%$ \\
\hline
\end{tabular}

Note. Sum of percentages exceed 100\% because some Tweets belonged in more than one theme.

\subsubsection{Theme 5: Monetary}

This theme included Tweets that are related to money such as profiteering or promoting a business. Some were trying to use the situation to promote their business or their twitter account through offering actual toilet rolls. Others were profiteering through selling toilet paper at a higher price. For example:

SHINY GIVEAWAY Retweet and Follow for a chance to win a full pack of shiny, Scott toilet paper rolls. Your local market ran out of them, but I haven't. Will pick 5 winners at the end of the week! Yup giveaways are back!!

\section{Discussion}

The Coronavirus 2019 pandemic has led to several unprecedented events in modern history, including the strictly enforced social distancing rules to prevent contagion, the tremendous disruption to countless businesses, and the panic buying phenomena observed across nations. The irrational hoarding behaviours could be explained by several theories. While fear is influenced by the perception of threat, the response to fear could be driven by emotions to maintain control over the ambivalence. Hoarding of food and necessities could offer temporary consolation to relieve anxiety and regain control, which often occurs in pandemics and crises [8]. During the 2003 SARS outbreak, panic buying perishable goods occurred in China and Hong Kong [20]. However, the scale of panic buying during the COVID-19 pandemic has been unparalleled. Our study of Tweets regarding panic buying of toilet paper by in vivo analysis examines psychology behind the behaviour.

Close to half of the Tweets expressed negative feelings towards the panic buying of toilet paper, such as anger and frustration over the insufficient stock of toilet rolls. The top three most influential Tweets had a total of 588,000 likes and 80,000 Retweets, all of which were negative, expressing confusion and anger towards panic buying. This could lead to the snowball effect in social media, where regular social media users could pick up sentimental feelings and opinions [10]. With rapid increase in digital connectedness, information is readily available and accessible via social media, replacing traditional media. This exacerbates the dissemination and transmission of psychological reactions in midst of public panics during pandemics [3, 21]. During the H1N1 outbreak in 2009, Twitter 
analysis studies have found misinformation could be easily spread through social media $[14,15]$. However, our study also identified positive Tweets, as a quarter of the Tweets contain supportive information regarding supplies of toilet paper and prompting others to stay calm in midst of the crisis. This might counterbalance the spread of anxiety and panic buying behaviours.

Our findings provided useful data to understand social psychology by comprehending public sentiments and opinions. This could equip nations around the world to be better prepared for another emergency crisis. Similar to crises during previous economic depressions, efforts from governments must be deployed to minimise underlying incentives of anxiety-induced panic buying. The government could utilise the social media, to monitor sentiments and address public concerns by issuing pertinent state-wise announcements to tackle mass panic and anxiety. This could efficiently ease the public concern of running out of daily necessities.

\section{Limitations}

Our analysis focused on text-based Tweets and did not analyse Tweets that were of pictures, pictures of texts, or videos. Review and analyses of Twitter media was outside of our research team's capacity due to manual retrieval and coding requirements. But a hand search and coding of a sample of media-based Tweets reviewed they were consistent with our themes. With the advancement in information technology, future research can more efficiently analyse attitudes, emotions and themes based on images and videos. For example, Google Cloud's Vision API have pre-trained machine learning models that can detect text and emotions from images, then code them into predefined categories.

The inferentiality of Tweets does not represent the totality across time. Tweets posted earlier may have more time to get Retweeted and liked initially, but then loses further interaction. Therefore, to reduce this bias, we collected the data with a lag time of a week. Our supplementary analyses confirmed there were no significant correlations between Tweet date and number of Retweets $(\mathrm{r}=0.01, p=.537)$ or Likes ( $\mathrm{r}=0.01, p=.631$; Figure S1).

The use of hashtags is not consistent across different populations. Certain cohorts and socio-demographic groups may differ in the use of hashtags in their posts. Therefore, to increase the generalisability of our findings, we included both 'toilet paper' and 'toilet roll' as search terms. In addition, $20 \%$ of Twitter's daily users are from the USA, and in general are younger and have higher socio-economic status [22]. Nevertheless, due to the large number of 145 million daily active users and 500 million posts per day, our findings represent conversations from a substantial number of people in the society.

\section{Conclusion}

In the modern epoch of technology, social media has become one of the most dominant tools of information dissemination and can be drawn upon to gain insights on population mental health. During the COVID-19 pandemic, anxiety-induced behaviours could be observed by the vast amount of social media posts regarding toilet paper buying. Our study found that while many people on Twitter expressed negative sentiments and frustration towards the panic buying of toilet paper, some Tweets providing supportive and positive information were quite influential as well. If researchers, relevant businesses or government agencies could utilise these readily accessible instruments, it could assist in examining public psychology and sentiments towards nationwide policies, to formulate appropriate strategies to ease public concerns. It would be worthwhile to further invest into social media analysis for research on public mental health.

\section{Declarations}

Supplementary Materials: The following are available online at http://doi.org/10.13140/RG.2.2.22158.38728, Figure S1: Scatterplots of Retweets and Likes by date of 
Tweets; Table S1: Most influential Tweets based on Retweets and Likes. Datasets generated are available online, https://doi.org/10.13140/RG.2.2.33547.03362.

Author Contributions: Conceptualization, J.L., G.C. and C.T.; methodology, V.C., J.L., and C.L.; software, J.L., J.C. and C.L.; validation, C.T. and J.C.; formal analysis, C.T. and J.C.; investigation, C.T. and J.C.; data curation, V.C., J.L., and C.L; writing-original draft preparation, J.L., J.C. and C.T.; writing-review and editing, J.C., G.C., V.C. and C.L.; visualization, J.L., J.C. and C.T.; supervision, G.C.; project administration, J.L. and G.C.. All authors have read and agreed to the published version of the manuscript.

Funding: This research received no external funding.

Code Availability: Data was collected using an automatic web crawler [19] with Python 3.7.3 on 8 April 2020. Manual coding process was conducted on NVivo by two independent researchers.

Conflicts of Interest: The authors declare no conflict of interest.

\section{References}

1. Chakraborty, N., The COVID-19 pandemic and its impact on mental health. Progress in Neurology and Psychiatry, 2020. 24(2): p. 21-24.

2. Dos Santos, C.F., M. Picó-Pérez, and P. Morgado, COVID-19 and Mental Health-What Do We Know So Far? Frontiers in Psychiatry, 2020.11.

3. Sim, K., et al., The anatomy of panic buying related to the current COVID-19 pandemic. Psychiatry research, 2020. 288: p. 113015113015.

4. Kirk, C.P. and L.S. Rifkin, I'll trade you diamonds for toilet paper: Consumer reacting, coping and adapting behaviors in the COVID-19 pandemic. Journal of Business Research, 2020. 117: p. 124-131.

5. Sheu, J.-B. and H.-T. Kuo, Dual speculative hoarding: A wholesaler-retailer channel behavioral phenomenon behind potential natural hazard threats. International Journal of Disaster Risk Reduction, 2020. 44: p. 101430.

6. Garfin, D.R., R.C. Silver, and E.A. Holman, The novel coronavirus (COVID-2019) outbreak: Amplification of public health consequences by media exposure. Health Psychol, 2020. 39(5): p. 355-357.

7. Scott, K.M., et al., Associations between lifetime traumatic events and subsequent chronic physical conditions: a cross-national, crosssectional study. PLoS One, 2013. 8(11): p. e80573.

8. Chen, C.Y., L. Lee, and A.J. Yap, Control Deprivation Motivates Acquisition of Utilitarian Products. Journal of Consumer Research, 2016. 43(6): p. 1031-1047.

9. Muchnik, L., S. Aral, and S.J. Taylor, Social influence bias: A randomized experiment. Science, 2013. 341(6146): p. 647-651.

10. Boulianne, S., Social media use and participation: a meta-analysis of current research. Information, Communication \& Society, 2015. 18(5): p. 524-538.

11. Ji, X., et al., Twitter sentiment classification for measuring public health concerns. Social Network Analysis and Mining, 2015. 5(1): p. 13.

12. Riordan, B.C. and K.B. Carey, Wonderland and the rabbit hole: A commentary on university students' alcohol use during first year and the early transition to university. Drug and alcohol review, 2019. 38(1): p. 34-41.

13. McClellan, C., et al., Using social media to monitor mental health discussions - evidence from Twitter. Journal of the American Medical Informatics Association, 2016. 24(3): p. 496-502.

14. Ahmed, W., et al., Novel insights into views towards H1N1 during the 2009 Pandemic: a thematic analysis of Twitter data. Health Information \& Libraries Journal, 2019. 36(1): p. 60-72.

15. Chew, C. and G. Eysenbach, Pandemics in the Age of Twitter: Content Analysis of Tweets during the 2009 H1N1 Outbreak. PLOS ONE, 2010. 5(11): p. e14118.

16. Miller, F., K. Davis, and H. Partridge, Everyday life information experiences in Twitter: a grounded theory. 2019.

17. Goldkuhl, G., Pragmatism vs interpretivism in qualitative information systems research. European journal of information systems, 2012. 21(2): p. 135-146.

18. Guest, G., E. Namey, and M. Chen, A simple method to assess and report thematic saturation in qualitative research. PLoS One, 2020. 15(5): p. e0232076.

19. Henrique, J., GetOldTweets-python. 2019.

20. Madson, M., Rhetoric of a Global Epidemic: Transcultural Communication about SARS: Huiling Ding. Carbondale, IL: Southern Illinois University Press, 2014. 325 pp. 2016, Taylor \& Francis.

21. Depoux, A., et al., The pandemic of social media panic travels faster than the COVID-19 outbreak. 2020, Oxford University Press.

22. Hughes, S.W.A. Sizing Up Twitter Users. 2020; Available from: https://www.shorturl.at/mqtS7. 